\title{
Influence of spin-orbit coupling on the transport properties of magnetic tunnel junctions
}

\author{
V. Popescu and H. Ebert \\ Department Chemie/Physikalische Chemie, Universität München, Butenandtstraße 5-13, D-81377 Munich, Germany \\ N. Papanikolaou \\ Institute of Microelectronics, NCSR "Demokritos", GR-15310 Aghia Paraskevi, Attiki, Greece \\ R. Zeller and P. H. Dederichs \\ Institut für Festkörperforschung, Forschungszentrum Jülich, Postfach 1913, D-52425 Jülich, Germany
}

(Received 30 May 2005; revised manuscript received 8 August 2005; published 22 November 2005)

\begin{abstract}
Using a fully relativistic implementation of the Landauer-Büttiker formalism, the ballistic conductance and magnetoresistance in the $\mathrm{Fe} / \mathrm{GaAs} / \mathrm{Fe}$ tunnel junction has been calculated. The underlying electronic structure of the system was obtained using a spin-polarized relativistic version of the screened Korringa-Kohn-Rostoker Green-function method. To analyze the influence of spin-orbit coupling induced spin-flip processes on the spin-dependent transport, a scheme introduced recently by us which allows one to split the relativistic conductance in terms of individual spin-diagonal and spin off-diagonal (spin-flip) components has been applied. Our previous investigations showed that a strong spin-flip channel is present in the conductance, having the most important influence for an antiparallel alignment of the magnetization in the two Fe leads. Motivated by these findings and based on model calculations in which the strength of the spin-orbit interaction has been manipulated we present a detailed analysis of the various features induced by the spin-orbit coupling inside the barrier, and how these modify the tunneling. We found that even in the ideal case of a specular interface the magnetoresistance can significantly drop if the spacer is characterized by a strong spin-orbit coupling.
\end{abstract}

DOI: 10.1103/PhysRevB.72.184427

PACS number(s): 75.70.Cn, 73.23.Ad, 71.70.Ej

\section{INTRODUCTION}

The last years witnessed an increased interest in investigating the spin-dependent transport between two ferromagnetic electrodes separated by either an insulator or a semiconductor, systems now commonly known as magnetic tunnel junctions (MTJs). While the first phenomenologically models for tunneling proposed by Jullière ${ }^{1}$ and Slonczewski ${ }^{2}$ connected the conductance dependence of MTJs with the densities of states and spin polarization of the leads and the transmission probability across the barrier, the first-principles based calculations of Butler, MacLaren, and co-workers ${ }^{3-5}$ revealed the importance of a more realistic treatment of the electronic structure of the junction as a whole. Since then, an impressive theoretical work followed, meant to give a reasonable description of this phenomenon. ${ }^{6}$ Recent investigations accounted for interface interdiffusion, ${ }^{7}$ considered the tunneling through a Schottky barrier, ${ }^{8}$ or made it possible to go beyond the linear-response regime. ${ }^{9}$

Despite these considerable efforts, the optimistic theoretical predictions based on $a b$ initio calculations are still not reached in corresponding experiments. Successful measurements of a sizeable magnetoresistance in Fe/GaAs/Fe MTJs (Ref. 10) did not find values larger than a few percent, not only far below the theoretical values but also much lower than those recorded for MTJs which use insulators as spacers. ${ }^{11}$ In their work, Zenger et al. ${ }^{10}$ also found a strong dependence of the magnetoresistance on the preparation conditions, ascribing its drop to an increased interface disorder. These findings could be reproduced by the theoretical calculations of Zwierzycki et l. $^{7}$ for the equivalent interface $\mathrm{Fe} / \mathrm{InAs}$, for which they found that even a small amount of interdiffused $\mathrm{Fe}$ atoms suffices to reduce significantly the spin asymmetry. This work demonstrated that a rather realistic geometry has to be used for theoretical investigations on the magnetoresistance to enable a sensible comparison between theory and experiment.

Experimental as well as theoretical works, on the other hand, emphasized the role spin-flip scattering processes might have in destroying the spin asymmetry. Most of the theoretical investigations, however, treated the two spin subsystems participating in the conduction independently (twocurrent model), introducing this way a spin filter. Published fully relativistic calculations treat the transport either on the basis of the Kubo-Greenwood ${ }^{12}$ or on the Landauer-Büttiker ${ }^{13}$ formalisms. A detailed analysis on how spin-orbit coupling - a pure relativistic effect-influences the conductance by mixing the different spin channels was until recently still missing.

In a previous work ${ }^{14}$ we have presented a fully relativistic formulation of the Landauer-Büttiker expression for conductance within the framework of screened Korringa-KohnRostoker Green-function method. We could show that the conductance can be separated, to a good accuracy and without leaving the relativistic representation, in spin diagonal and spin off-diagonal (spin-flip) components, a scheme enabling one to investigate in detail the effects induced by the spin-orbit coupling, qualitatively as well as quantitatively. Application of our formalism to the $\mathrm{Fe} / \mathrm{GaAs} / \mathrm{Fe}$ trilayer systems showed that spin-flip contributions to the conductance are important, especially in the case of an antiparallel alignment of the magnetization in the Fe leads where they are about one order of magnitude larger than the spindiagonal terms. 
In the present calculations of spin-dependent tunneling we extend our discussion to the qualitative and quantitative changes induced by accounting for the spin-orbit coupling inside the tunneling barrier. For this purpose, we perform model calculations in which the strength of the spin-orbit interaction is manipulated according to a scheme suggested some years ago, ${ }^{15}$ ignoring, however, any influences related to imperfections in the metal-semiconductor interface. In turn, the changes in the electronic structure of the semiconducting spacer induced by spin-orbit coupling and thus its role in assisting the tunneling through the barrier is investigated in some detail. For reasons mentioned above, the perfect geometry assumed for our calculations makes a direct comparison with the available experimental findings rather difficult. It will be shown, however, that even in a ballistic treatment with a perfect geometry the spin-orbit coupling in the barrier can cause significant changes in the magnetoresistance.

\section{THEORETICAL FRAMEWORK}

The electronic ground state and the transport properties have been determined on the basis of the screened KorringaKohn-Rostoker Green-function method (tight-binding KKR) combined with the decimation technique for twodimensional (2D) systems. ${ }^{16}$ Within this scheme, the oneelectron retarded Green function $G^{+}\left(\vec{r}, \overrightarrow{r^{\prime}} ; E\right)$ at energy $\varepsilon$ $=E+i \delta$ is expressed in terms of the so-called structural Green-function (GF) matrix $\underline{G}^{n n^{\prime}}(\varepsilon)$, describing the propagation between the sites $n, n^{\prime}$ located at $\vec{R}_{n}, \vec{R}_{n^{\prime}}$. The fully relativistic implementation of the screened KKR-GF method used in our present investigations has been achieved in a similar way as it was done before for the conventional KKR method for three-dimensional periodic systems. ${ }^{17}$ This leads to a very general and flexible scheme which allows one to account for spin polarization and all relativistic effectsincluding spin-orbit coupling (SOC)—on equal footing.

\section{A. Single-site Dirac equation}

To allow for a fully relativistic treatment means in particular that the basic single-site problem is solved for the Dirac Hamiltonian: ${ }^{17,18}$

$$
\widehat{\mathcal{H}}_{D}=c \vec{\alpha} \vec{p}+\beta m c^{2}+V_{\text {eff }}(\vec{r}),
$$

with the conventional Dirac matrices $\vec{\alpha}$ and $\beta,{ }^{18}$ and the effective potential $V_{\text {eff }}(\vec{r})$ consisting of a spin-independent potential $V(\vec{r})$ and a spin-dependent one $V_{\text {spin }}(\vec{r})$. Within the LSDA the latter behaves like a magnetic field that couples only to the electron spin degree of freedom, $V_{\text {spin }}(\vec{r})$ $=\beta \vec{\sigma} \vec{B}(\vec{r})$. Assuming further that the potential is spherically symmetric-muffin tin (MT) or atomic sphere approximation (ASA) - the Dirac Hamiltonian takes on the form ${ }^{17}$

$$
\widehat{\mathcal{H}}_{D}=c \vec{\alpha} \vec{p}+\beta m c^{2}+V(r)+\beta \sigma_{z} B_{\text {eff }}(r) .
$$

The regular and irregular solutions, $R_{\Lambda}(\vec{r}, E)$ and $H_{\Lambda}(\vec{r}, E)$, respectively, of the single-site Dirac equation have the general form

$$
\Psi_{\Lambda}(\vec{r}, E)=\sum_{\Lambda^{\prime}}\left(\begin{array}{c}
g_{\Lambda^{\prime} \Lambda}(r, E) \chi_{\Lambda^{\prime}}(\hat{r}) \\
i f_{\Lambda^{\prime} \Lambda}(r, E) \chi_{-\Lambda^{\prime}}(\hat{r})
\end{array}\right) .
$$

Here $\Lambda$ and $-\Lambda$ stand for $(\kappa, \mu)$ and $(-\kappa, \mu)$, respectively, with $\kappa$ and $\mu$ the spin-orbit and magnetic quantum numbers, while $\chi_{\Lambda}(\hat{r})$ are the spin-angular functions: ${ }^{18}$

$$
\chi_{\Lambda}(\hat{r})=\sum_{m_{s}= \pm 1 / 2} C_{\Lambda}^{m_{s}} Y_{l}^{\mu-m_{s}}(\hat{r}) \chi_{m_{s}},
$$

with $C_{\Lambda}^{m_{s}} \equiv C\left(l, 1 / 2, j ; \mu-m_{s}, m_{s}\right)$ the Clebsch-Gordon coefficients, $Y_{l}^{\mu-m_{s}(\hat{r})}$ the complex spherical harmonics and $\chi_{m_{s}}$ the Pauli spinors.

\section{B. A relativistic expression for the conductance}

Within the linear-response theory, i.e., ignoring nonlinear effects caused by a finite bias voltage applied to the system, Baranger and Stone ${ }^{19}$ have shown that the ballistic conductance between two leads can be expressed as the flux of the conductivity tensor $\underline{\sigma}\left(\vec{r}, \vec{r}^{\prime}\right)$ into these leads:

$$
g=-\int_{C} d^{2} r \int_{C^{\prime}} d^{2} r^{\prime} \vec{n} \underline{\sigma}\left(\vec{r}, \overrightarrow{r^{\prime}}\right) \overrightarrow{n^{\prime}},
$$

with $\vec{n}, \vec{n}^{\prime}$ unit vectors normal to the $C, C^{\prime}$ surfaces within the two leads. The conductivity tensor is given by ${ }^{20}$

$$
\underline{\sigma}\left(\vec{r}, \vec{r}^{\prime}\right)=-\frac{\hbar}{4 \pi} \sum_{p, q= \pm 1}(p q) \operatorname{Tr}\left[\vec{j}(\vec{r}) G\left(\vec{r}, \vec{r}^{\prime} ; \varepsilon_{p}\right) \vec{j}\left(\overrightarrow{r^{\prime}}\right) G\left(\overrightarrow{r^{\prime}}, \vec{r} ; \varepsilon_{q}\right)\right],
$$

where $\vec{j}(\vec{r})$ is the current density operator and $\varepsilon_{p}=E_{F}+p \mathrm{i} 0$, with $E_{F}$ the Fermi energy of the system. Combining the last two equations leads to

$$
\begin{aligned}
g= & -\frac{\hbar}{2 \pi} \operatorname{Tr} \int_{C} d^{2} r \int_{C^{\prime}} d^{2} r^{\prime}\left[(\vec{j}(\vec{r}) \cdot \vec{n}) G\left(\vec{r}, \vec{r}^{\prime} ; \varepsilon_{F}\right)\right. \\
& \left.\times\left(\vec{j}\left(\vec{r}^{\prime}\right) \cdot \vec{n}^{\prime}\right) G\left(\overrightarrow{r^{\prime}}, \vec{r} ; \varepsilon_{F}^{*}\right)\right] .
\end{aligned}
$$

For the free-electron case Baranger and Stone also could prove that their result is equivalent to the Landauer-Büttiker conductance formula. ${ }^{21}$ More recently, Mavropoulos et al. ${ }^{22}$ extended the proof to the case of Bloch electrons, giving a corresponding expression within the framework of screened KKR method. Based on their derivation, to which we refer for further details and notation, we could show ${ }^{14}$ that a fully relativistic generalization can be straightforwardly achieved. The ballistic conductance between two layers (atomic planes) $I$ and $I^{\prime}$ in a $2 \mathrm{D}$ system is given by

$$
g=\frac{e^{2}}{h} \frac{1}{A_{\mathrm{SBZ}}} \int_{\mathrm{SBZ}} d^{2} k_{\|} g\left(\vec{k}_{\|}\right)
$$

with $g\left(\vec{k}_{\|}\right)$the $\vec{k}_{\|}$-resolved transmission probability, 


$$
g\left(\vec{k}_{\|}\right)=\sum_{\substack{\alpha \in I \\ \alpha^{\prime} \in I^{\prime}}} \operatorname{Tr}\left[\underline{J}^{n}\left(\varepsilon_{F}^{*}, \varepsilon_{F}\right) \underline{G}^{n n^{\prime}}\left(\vec{k}_{\|}, \varepsilon_{F}\right) \underline{J}^{n^{\prime}}\left(\varepsilon_{F}, \varepsilon_{F}^{*}\right) \underline{G}^{n n^{\prime} \dagger}\left(\vec{k}_{\|}, \varepsilon_{F}\right)\right],
$$

and $\alpha$ the index of the atomic site with position vector $\vec{r}_{\alpha}$ in the $\nu$ th 2D unit cell of layer $I$, such that $\vec{R}_{n}=\vec{R}_{I}+\vec{\chi}_{\nu}+\vec{r}_{\alpha}$. Here $\underline{G}$ and $\underline{J}$ are matrices labeled by the relativistic quantum numbers, i.e., $(\underline{A})_{\Lambda \Lambda^{\prime}}=A_{\Lambda \Lambda^{\prime}}$, and they represent, respectively, the structural Green-function matrix and the matrix of the $z$ component of the relativistic current-density operator. ${ }^{14}$

Using a relativistic expression for conductance implies, on one hand, that the SOC is explicitly taken into account in both of these quantities. This by no means rules out the possibility of treating the SOC within perturbation theory, provided that the spin-orbit interaction is small. A direct inclusion, as it is done here, is, however, more general.

Within a fully relativistic formalism, on the other hand, the electron spin is not a constant of motion and a standard interpretation of the transport in terms of individual spin contributions is no longer possible. We could remove this problem by suggesting a scheme which allows one to split the relativistic conductance into spin-diagonal and spin offdiagonal terms. ${ }^{14}$ This is based on the exact unitary transformation between the relativistic $\Lambda=(\kappa, \mu)$ and the nonrelativistic $\mathcal{L}=\left(l, m_{l}, m_{s}\right)=\left(L, m_{s}\right)$ representation provided by the Clebsch-Gordon coefficients matrix $:^{23}$

$$
\mathcal{A}_{\mathcal{L} \mathcal{L}^{\prime}}=\sum_{\Lambda \Lambda^{\prime}} S_{\Lambda \mathcal{L}^{A}} A_{\Lambda \Lambda^{\prime}} S_{\mathcal{L}^{\prime} \Lambda^{\prime}}^{\dagger}
$$

Expanding the trace in Eq. (9) and neglecting the terms connected with the spin off-diagonal terms in the current density matrix $\mathcal{J}$ one arrives at a sum over spin quantum numbers $m_{s}$ and a trace over the nonrelativistic quantum numbers $L$. One can define this way the spin-decomposed conductance terms $\widetilde{g}^{m_{s} m_{s}^{\prime}}$ through

$$
\widetilde{g}^{m_{s} m_{s}^{\prime}} \propto \operatorname{tr}\left[\underline{\mathcal{J}}^{n, m_{s} m_{s}} \underline{\mathcal{G}}^{n n^{\prime}, m_{s} m_{s}^{\prime}} \underline{\mathcal{J}}^{n^{\prime}, m_{s}^{\prime} m_{s}^{\prime}} \underline{\mathcal{G}}^{n n^{\prime}, m_{s}^{\prime} m_{s}^{\dagger} \dagger}\right] .
$$

Thus the exact relativistic expression for conductance, Eq. (8), can be approximated by the sum over individual spindiagonal and spin-flip contributions $\widetilde{g}^{m_{s} m_{s}^{\prime}}$ in a $\vec{k}_{\|}$-resolved as well as $\vec{k}_{\|}$-integrated formulation

$$
g \approx \widetilde{g}=\sum_{m_{s}, m_{s}^{\prime}} \widetilde{g}^{m_{s} m_{s}^{\prime}} .
$$

It is important to note here that this approximate expression will be further used only to analyze qualitatively the effect of including the SOC in the calculations. Neglecting the spin off-diagonal elements of the current density matrix is not a severe approximation since these are three to four orders of magnitude smaller than the spin-diagonal ones. The major influence of the SOC is still present through the structural Green-function matrix $\underline{\mathcal{G}}$, thus spin-flip processes occurring as a result of the scattering between the two leads are exactly accounted for.

\section{Spin-orbit coupling manipulation}

In order to investigate quantitatively the effect of SOC, we will present several results of model calculations that have been performed using a scheme suggested earlier. ${ }^{15}$ This approach allows one to gradually switch the spin-orbit interaction strength without leaving the relativistic representation. For the sake of reference, we review in the following derivation of the basic formulas.

The spin-angular functions $\chi_{\Lambda}(\hat{r})$, Eq. (4), are eigenfunctions of the spin-orbit operator

$$
\begin{gathered}
\hat{K}=\vec{\sigma} \cdot \hat{\vec{L}}+1, \\
\hat{K} \chi_{\Lambda}(\hat{r})=-\kappa \chi_{\Lambda}(\hat{r}) .
\end{gathered}
$$

Scaling of the spin-orbit interaction strength implies to multiply the term $\vec{\sigma} \cdot \hat{\vec{L}}$ with a factor $x$. In spite of this scaling, the functions $\chi_{\Lambda}(\hat{r})$ are still eigenfunctions of the modified spinorbit operator $\hat{K}_{x}$ :

$$
\begin{gathered}
\hat{K}_{x}=x \vec{\sigma} \cdot \hat{\vec{L}}+1, \\
\hat{K}_{x} \chi_{\Lambda}(\hat{r})=-\kappa_{x} \chi_{\Lambda}(\hat{r}), \quad \kappa_{x}=-1+x(1+\kappa) .
\end{gathered}
$$

As one can see, for $x=1$ nothing changes, while for $x=0$ one gets $\kappa_{x}=-1$, i.e., the value of the spin-orbit quantum number of the $s$ states without spin-orbit interaction. Consequently, replacing $\kappa$ with $\kappa_{x}$, one is switching off the spin-orbit interaction of any partial wave function setting $x=0$, while other values allow one to modify its strength.

One has to note here that this transformation has been done by rewriting the set of coupled radial Dirac equations. ${ }^{15}$ In this way, although still working in the relativistic $\Lambda$ representation, the minor component becomes meaningless, and the ansatz

$$
\Psi_{\Lambda}(\vec{r}, E)=\sum_{\Lambda^{\prime}} g_{\Lambda^{\prime} \Lambda}(\vec{r}, E) \chi_{\Lambda^{\prime}}(\hat{r})
$$

has to be used instead of Eq. (3) in solving the single-site problem.

\section{RESULTS AND DISCUSSION}

\section{A. Computational details}

As a case study for our relativistic implementation we have chosen an As-terminated $\mathrm{Fe} / n(\mathrm{GaAs}) / \mathrm{Fe}$ system with the parent lattice of the zinc-blende structure having the lattice constant $a=5.6536 \AA$. Because our investigations focused on the effect of SOC inside the spacer, all the other effects connected with interface characteristics, such as termination (As or $\mathrm{Ga}$ ), interdiffusion, disorder, or lattice relaxation have been ignored. A collinear magnetic configuration has been considered, with the magnetization along the interface normal. The calculations have been performed for two different cases, a parallel (P) and an antiparallel (AP) alignment of the magnetization in the two Fe leads.

In a first step, the electronic structure has been determined self-consistently within the LSDA, with the parametrization 
for the exchange-correlation potential proposed by Vosko, Wilk, and Nussair, ${ }^{24}$ making use of the atomic sphere approximation (ASA) and the angular-momentum expansion truncated at $l_{\max }=2$. The space filling required by ASA has been achieved by inserting empty spheres in the zinc-blende structure, ending up with two atomic sites in the 2D unit cell. For the SCF calculations the junction was modeled by a $6 \mathrm{Fe} / 9(\mathrm{GaAs}) / 5 \mathrm{Fe}$ slab sandwiched between two semiinfinite Fe leads. As it has been found also by other authors for similar systems, ${ }^{25,26}$ this slab thickness suffices to obtain for the middle GaAs layers an essentially semiconductor bulklike potential.

For the spin-dependent transport investigations the semiconductor thickness has been varied between $n=21$ and $n$ $=61$ atomic layers. A frozen potential model-as used, e.g., by Mavropoulos et al. ${ }^{26}$ - has been adopted to describe these thick junctions. Assuming the geometry of the trilayer described as

$$
6 \mathrm{Fe} / 4(\mathrm{GaAs}) /(2 m-1) \mathrm{GaAs} / 4(\mathrm{GaAs}) / 5 \mathrm{Fe},
$$

with $m=(n-7) / 2$, one uses the potentials for the Fe leads and for the $\mathrm{Fe} / \mathrm{GaAs}$ interface as obtained from the $\mathrm{SCF}$ calculations for the small junction, while for all the $2 m+1$ inner $\mathrm{Ga}$ and As atoms the bulklike SCF potentials of the atoms in the middle of the $\mathrm{Fe} / 9(\mathrm{GaAs}) / \mathrm{Fe}$ junction are taken over. The angular momentum cutoff for the transport calculations was set to $l_{\max }=3$. The Fermi energy of the system is that determined by the semi-infinite Fe leads, lying in the middle of the gap (occurring in the inner semiconductor layers). In contrast to Ref. 26, where the spin injection was studied, an extra potential shift, meant to mimic the gate voltage, has not been applied to the inner potentials. Thus the results to be presented below correspond to a zero-bias tunneling conductance regime. For each of the spacer thicknesses the calculations have been performed in the two magnetic configurations $\mathrm{P}$ and $\mathrm{AP}$, which allows us to determine the tunneling magnetoresistance (TMR) ratio defined as

$$
T=\frac{g^{\mathrm{P}}-g^{\mathrm{AP}}}{g^{\mathrm{P}}} .
$$

\section{B. Fully relativistic calculations for the $\mathrm{Fe} / \mathrm{GaAs} / \mathrm{Fe}$ trilayer system}

The mechanisms governing the ballistic tunneling are thoroughly discussed in the literature..$^{3,27,28}$ Among them, the symmetry of the electronic states of the ferromagnetic leads and their coupling to the evanescent states and their decay in the semiconductor, the resonant interfacial states present in the minority spin channels, as well as the character of chemical bonding at the metal-semiconductor interface were considered to be of particular importance.

We have already presented ${ }^{14}$ results of calculations based on the formalism sketched in Sec. II, obtained for Asterminated $\mathrm{Fe} / n(\mathrm{GaAs}) / \mathrm{Fe}$ junctions. In order to extend the discussion on the effect of the SOC on the transport properties of this system, we first summarize our previous findings, referring to Figs. 1 and 3 of Ref. 14.
For the conductance, calculated by means of the relativistic expression, Eq. (8), an essentially exponential decay with the spacer thickness was obtained, for both $\mathrm{P}$ and AP magnetic configurations. The corresponding TMR ratio exhibits an abrupt increase for thicker spacers. An analysis of the spin-decomposed conductance as obtained from Eqs. (11) and (12) revealed that, in line with previous, nonrelativistic results, the decay rates of the majority $\left(\widetilde{g}^{\downarrow \downarrow}\right) \mathrm{P}$, minority $\left(\widetilde{g}^{\Uparrow \uparrow}\right)$ $\mathrm{P}$, and majority or minority AP terms strongly differ from one another, with majority $\mathrm{P}$ representing the slowest decay channel. These characteristics have been related, in tunneling ${ }^{3}$ as well as in injection-detection ${ }^{26}$ regimes, to the interplay between the coupling efficiency of different states across the interface and their decay in the barrier. We note here that, due to the chosen magnetic configuration, the symmetry aspects discussed in the cited papers are essentially the same. The only difference is that the electronic states involved have merely a certain degree of spin polarization rather than a pure spin character.

For the $\mathrm{P}$ alignment the results did not sensibly differ from what one would get in a nonrelativistic calculation: the conductance is overwhelmingly dominating in the majority channel, while the spin-flip term $\left(\widetilde{g}^{\uparrow \downarrow}+\widetilde{g}^{\downarrow \uparrow}\right)$ makes the second important contribution to the total conductance. In the AP alignment, on the other hand, we could find that the main contribution (around 80\%) comes from the spin-flip term, that would be missing if one performed a nonrelativistic calculation. This term is also characterized by a different decay rate in the two magnetic configurations. In the $\mathrm{P}$ alignment it is nearly identical to that of the minority term. In the AP alignment on the other hand, it is smaller than the spindiagonal terms, which suggests a kind of spin-orbit assisted tunneling process inside the barrier.

Further qualitative effects of the SOC can be observed by inspecting the results presented in the top panel of Fig. 1. Here the distribution of the conductance is shown over the individual channels around the central point $\bar{\Gamma}$ in the twodimensional Brillouin zone (2DBZ) for the P (left panel) and AP (right panel) alignment, as calculated using the relativistic expression Eq. (9), for different thicknesses representative for the investigated range.

Several already known and discussed characteristics ${ }^{3,28}$ can be recognized in Fig. 1: the pronounced peak at the center of the 2DBZ in the $\mathrm{P}$ alignment which becomes narrower for thicker spacer or the resonant peaks out of the normal incidence in both alignments, stemming from the localized minority states. One notes, however, that the structure of $g\left(\vec{k}_{\|}\right)$in the AP case tends to become the same as for $\mathrm{P}$ as the thickness of the semiconductor increases, whereas previous calculations, based on a nonrelativistic treatment, always found a local minimum in $g^{\mathrm{AP}}\left(\vec{k}_{\|}\right)$at the $\bar{\Gamma}$ point.

This "hole" near the normal incidence for minority $\mathrm{P}$ and in the AP alignment is usually ascribed to the lack of minority states having the proper symmetry to enable the coupling with the evanescent states in the spacer. It is obvious that the inclusion of the SOC relaxes this selection rule and a significant transmission occurs around the $\bar{\Gamma}$ point also in the AP alignment.

When moving away from the center of the 2DBZ, the transmission shows an oscillatory behavior. This peculiarity, 

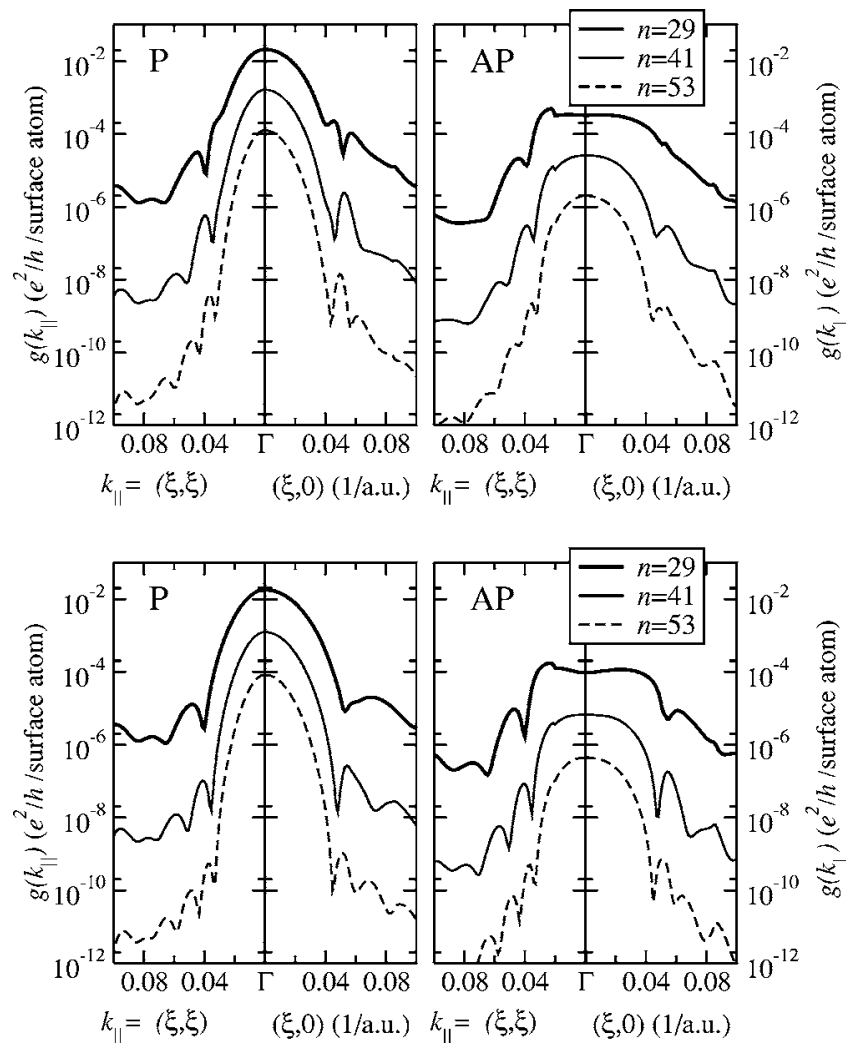

FIG. 1. $\vec{k}_{\|}$-resolved relativistic transmission probability $g\left(\vec{k}_{\|}\right)$, as given by Eq. (9), in $\mathrm{Fe} / n(\mathrm{GaAs}) / \mathrm{Fe}$ along the (110) and (100) directions of the zinc-blende structure, for different numbers of layers $n$. Top: exact SOC calculations. Bottom: SOC switched off on the 2m-1 Ga and As atoms inside the spacer (see text). Left and right side of each panel correspond, respectively, to a parallel (P) and antiparallel (AP) alignment of the magnetization in the two $\mathrm{Fe}$ leads.

as discussed already by MacLaren et al. ${ }^{5}$ arises from the interference of the electron waves inside the barrier. We will show in the next subsection, by means of model calculations in which the spin-orbit interaction is manipulated within the inner GaAs layers, that also this process is influenced by SOC.

\section{Spin-orbit coupling effect on the electronic structure of GaAs}

The evanescent states in the semiconducting or insulating barrier-so-called metal-induced gap states (MIGSs) - have been recognized quite early as having an important role in spin-dependent tunneling. Mavropoulos et al. ${ }^{29}$ calculated the complex band structure of several direct and indirect gap semiconductors, deriving from it the decay parameter, identified as the imaginary part $\kappa_{z}$ of the complex wave vector normal to the surface. Making the important observation that the complex energy bands inherit the symmetry of the real ones they are connecting, they found that direct band-gap semiconductors should have a minimum decay rate at the center of the 2DBZ. Later on, Butler et al. ${ }^{28}$ found that this applies also to $\mathrm{MgO}$, i.e., an insulating barrier. For this mag- netic tunneling junction these authors discussed in detail the interference of tunneling states showing that the aforementioned oscillations in $g\left(\vec{k}_{\|}\right)$are also resulting from the complex band structure in the energy gap. From the $\vec{k}_{\|}$dependence of $\kappa_{z}$ two different transmission regimes could be identified: in the first of them, centered around the $\bar{\Gamma}$ point, the transmission decays as a sum of exponentials while in the second one it is a damped oscillatory function of thickness. It is noteworthy to mention that the transition from one of the regimes to the other depends on the thickness of the barrier, as it can be also seen in Fig. 1. Finally, in discussing the role of the interfacial resonant states, Butler et $a .^{28}$ as well as Mathon and Umerski ${ }^{27}$ could show why these hardly contribute to the tunneling for relatively thick spacers. For symmetry reasons, they are able to couple only to fast decaying evanescent states in the barrier. All these findings have shown that the complex band structure is of particular importance in explaining various features of tunneling.

We will discuss in the following the effect of SOC on the GaAs band structure (real and complex) and how it may influence the magnetoresistance of the $\mathrm{Fe} / \mathrm{GaAs} / \mathrm{Fe}$ trilayer system. The complex energy bands are calculated using a similar procedure as in Ref. 29: the $\vec{k}$ vector is split into a part $\vec{k}_{\|}$parallel to the surface of interest and a part perpendicular to it, $q_{z}=k_{z}+\mathrm{i} \kappa_{z}$. For each real $\vec{k}_{\|}$the dispersion relation $E=E\left(q_{z}\right)$ can be determined. Fixing the energy $E$ instead and scanning the 2DBZ one can calculate the decay parameter $\kappa_{z}\left(\vec{k}_{\|}\right)$.

Calculations have been performed for the threedimensional zinc-blende unit cell using the $\mathrm{Ga}$, As, and empty sphere potentials of the inner layers of the modeled tunnel junction, thus the Fermi energy lying in the middle of the gap. The results for the real energy bands near the $\Gamma$ point for $\vec{k}_{\|}=0$ along the $k_{z}$ direction and for the complex energy bands at $k_{z}=0$ are presented in the top panel of Fig. 2 . The full lines represent the exact relativistic calculations, i.e., using the nonmanipulated spin-orbit coupling operator, Eq. (13), while the dashed lines denote energy bands obtained switching off the SOC on the Ga and As sites, i.e., using for both of these atoms the modified SOC operator, Eq. (15) with $x=0$. For the latter case, which would correspond to a scalar-relativistic calculation, the Fermi energy has not been recalculated, the effect of SOC on the center of gravity of the electronic bands being negligibly small. ${ }^{15}$ As expected, the most evident feature that can be seen in this figure is the lifting of degeneracy in the valence band (VB) due to inclusion of SOC. This is a direct consequence of the fact that the top of this band has dominantly As $p$ character, while the bottom of the conduction band (CB), where no differences between the two types of calculation can be noticed, has mainly Ga $s$ character.

One further notes that the closed-loop complex band at $k_{z}=0$ connecting the top of the VB with the bottom of the CB should favor a spin-mixing in transmission, because the high VB $p_{3 / 2}$ states lack a well defined spin character, as it is the case for $p_{1 / 2}$ and $s_{1 / 2}$ states. Moreover, in the exact SOC case $(x=1)$, this loop is somewhat narrower as in the SOC off ( $x=0)$ case.

The lifting of the degeneracy by inclusion of the SOC and the narrowing of the closing loop is also reflected in the 


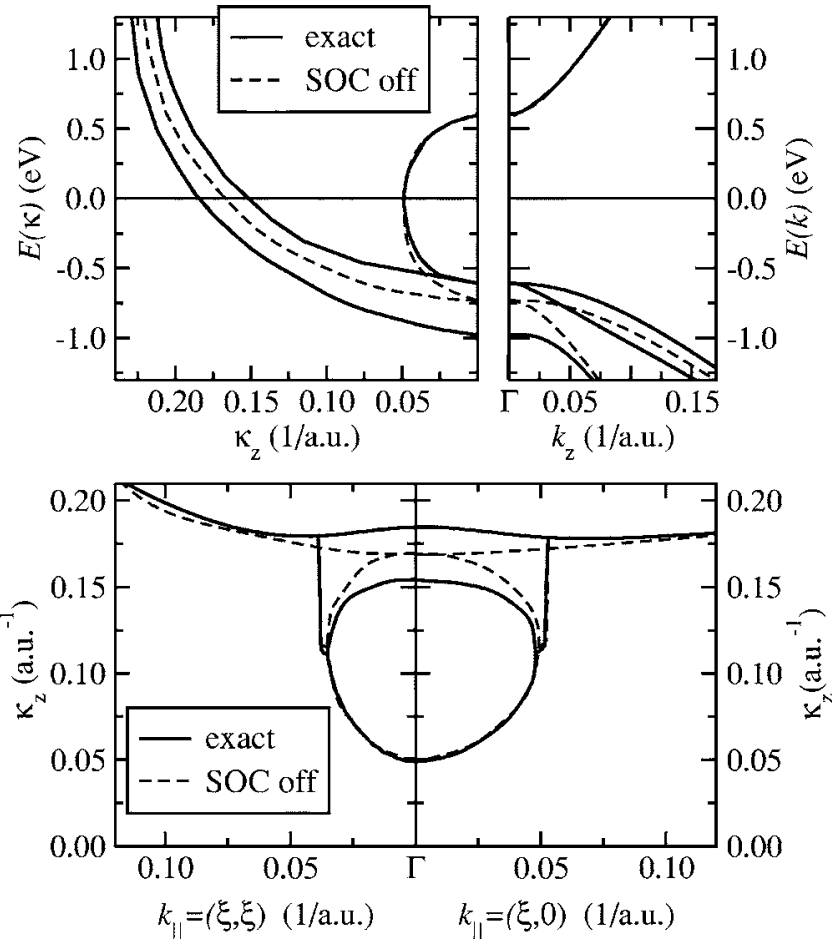

FIG. 2. Top: energy bands near the $\Gamma$ point obtained using the potentials of the bulklike GaAs middle layers in $\mathrm{Fe} / \mathrm{GaAs} / \mathrm{Fe}$ for the complex vector $q_{z}=k_{z}+\mathrm{i} \kappa_{z}$. The energy zero corresponds to the Fermi energy of the Fe leads. Bottom: dependence of the imaginary part $\kappa_{z}$ on $\vec{k}_{\|}$as deduced from the complex energy bands at the Fermi energy $E_{F}$ for $k_{z}=0$. Solid lines: exact relativistic calculations; dashed lines: SOC switched off on As and Ga sites, without recalculating the Fermi energy.

decay parameter $\kappa_{z}\left(\vec{k}_{\|}\right)$. This has been calculated for $k_{z}=0$ at the energy corresponding to the middle of the gap and it is shown in the bottom panel of Fig. 2 for two different directions in the Brillouin zone. As one can see, around the $\Gamma$ point three distinct branches are occurring when the full SOC is included $(x=1)$. The lowest one of them, corresponding to the strongest transmission, originates from the abovementioned $p_{3 / 2}$ states. The $\vec{k}_{\|}$points where the first two branches join lie closer to the $\Gamma$ point when SOC is accounted for. We recall that these points have been identified by Butler et al. ${ }^{28}$ as marking the transition between two different regimes of decay in the transmission probability.

Obviously, these qualitative differences induced by SOC in the electronic structure of GaAs (or other similar spacers) should also be reflected in the transport properties of the magnetic junction. The whole process is of course more complex because of the SOC being present in addition in the ferromagnetic leads and at the interface. The former case has been discussed, for example, by Ebert. ${ }^{17}$ The latter, on the other hand, involving the coupling across the metalsemiconductor interface and changes in the interfacial states, needs a separate discussion which will be presented elsewhere. ${ }^{30}$ We will therefore restrict ourselves in the following to the effect of SOC inside the barrier.

\section{Spin-orbit coupling manipulation inside the GaAs barrier}

Based on the frozen-potential model for the tunnel junction, we performed calculations for the conductance in which the SOC has been suppressed for the inner $(2 m-1) \mathrm{Ga}$ and As atoms of the $\mathrm{Fe} / n \mathrm{GaAs} / \mathrm{Fe}$ trilayer system, with $m=(n$ -7)/2 (see above). For similar arguments as given before for the electronic structure of bulklike GaAs, the Fermi energy, fixed by the semi-infinite Fe leads, has not been recalculated. In other words, since the bottom of the CB is not influenced by the presence of SOC, the height of the barrier does not change in the two cases, i.e., taking $x=0$ or $x=1$ in Eq. (15).

Results for the $\vec{k}_{\|}$-resolved transmission probability calculated with $x=0$ are presented in the bottom panel of Fig. 1, for the same thicknesses as in the full SOC case (top panel). One can see that suppressing the SOC inside the barrier changes $g\left(\vec{k}_{\|}\right)$across the junction in an appreciable way. These changes are more pronounced for the AP alignment, as it could be expected from the results discussed in the previous subsection. Indeed, the transport in the P alignment being dominated by the majority channel, the transmission through the barrier is hardly modified by the SOC. Noteworthy quantitative changes can be seen only close to the normal incidence. Nevertheless, as $k_{\|}$increases, one sees a noticeable change in the oscillatory profile of $g\left(\vec{k}_{\|}\right)$, especially along the $k_{x}=0$ (and $k_{y}=0$ ) direction. The same is true for the AP alignment, but here the differences are more pronounced. Moreover, for this magnetic configuration the transmission is strongly reduced also around the $\bar{\Gamma}$ point when the SOC is suppressed. Last but not least, comparing the two different alignments $\mathrm{P}$ and AP for each of the cases $x=0$ and $x=1$, respectively, one notes that, with SOC suppressed inside the barrier, the peaks in $g\left(\vec{k}_{\|}\right)$occur around the same points of the 2DBZ and have comparable amplitudes. For the full SOC calculations, on the other hand this is no longer true; thus the configuration dependent interference pattern supplies another indication for a spin-orbit coupling assisted tunneling within the spacer. This interpretation is also supported by Fig. 3 which shows the spin decomposition of the transmission probability $\widetilde{g}^{m_{s} m_{s}^{\prime}\left(\vec{k}_{\|}\right)}$in the AP alignment, as given by Eq. (11). Here, results of exact relativistic calculations (full lines) are compared with those of SOC-manipulated ones (dashed lines) for the same thicknesses as in Fig. 1. It can be clearly seen that the spin-diagonal term $g^{\downarrow \downarrow}\left(\vec{k}_{\|}\right)$(left) is much less influenced by SOC than the spin-flip one $g^{\uparrow \downarrow}\left(\vec{k}_{\|}\right)$(right). One finds a relatively small (roughly 10\%) thickness dependent reduction and no changes in the oscillations of $g^{\downarrow \downarrow}\left(\vec{k}_{\|}\right)$when the SOC is suppressed. For the spin-flip term in turn, the SOC suppression is accompanied by a significant reduction in transmission (one order of magnitude and more) which shows no thickness dependence, and a clear change in the interference pattern. It should be emphasized here that this does not minimize the role of the SOC acting within the rest of the junction, since the remaining $g^{\uparrow \downarrow}\left(\vec{k}_{\|}\right)+g^{\downarrow \uparrow}\left(\vec{k}_{\|}\right)$term for $x=0$ arises solely from the spin-flip scattering along the leads and the interfaces, where SOC has not been manipulated for the present model calculations. Thus the dominating SOC effect originates from these two regions. 


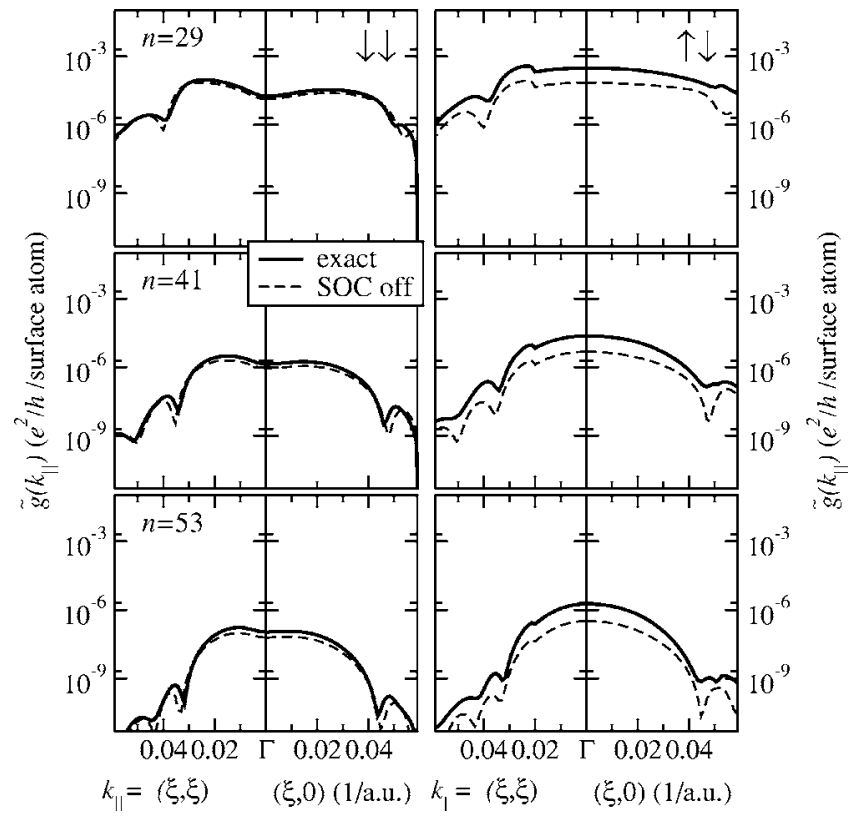

FIG. 3. Spin-decomposed terms $\widetilde{g}^{m_{s} m_{s}^{\prime}}\left(\vec{k}_{\|}\right)$of the $\vec{k}_{\|}$-resolved transmission probability in $\mathrm{Fe} / n(\mathrm{GaAs}) / \mathrm{Fe}$ along the (110) and (100) directions of the zinc-blende structure, as obtained from Eq. (11), for several thicknesses $n$ in the AP alignment. Left and right panel show, respectively, the spin-diagonal, $\widetilde{g}^{\downarrow} \downarrow\left(\vec{k}_{\|}\right)$, and the spinflip, $\widetilde{g}^{\uparrow} \downarrow\left(\vec{k}_{\|}\right)+\widetilde{g}^{\downarrow \uparrow}\left(\vec{k}_{\|}\right)$, contributions to the conductance. Thick lines: exact SOC calculations; dashed lines: SOC switched off within the 2m-1 inner GaAs layers, without recalculating the Fermi energy.

The conductance and the TMR of the Fe/GaAs/Fe trilayer system change accordingly, as a result of all of the effects discussed above. Figure 4 shows for the $\mathrm{P}$ as well as for the AP alignment that compared to the full relativistic calculations (results from Fig. 1 of Ref. 14 are replotted here with lines), the conductance (left panel of Fig. 4) decreases when SOC is suppressed inside the junction, with the reduction being more pronounced in the AP case. The different

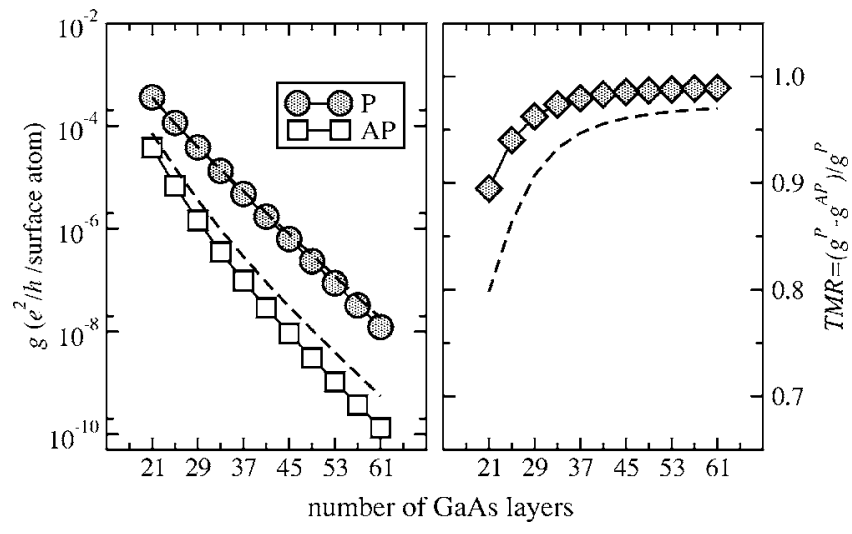

FIG. 4. Left: tunneling conductance for $\mathrm{Fe} / n(\mathrm{GaAs}) / \mathrm{Fe}$ trilayer system [for parallel (P) and antiparallel (AP) alignment] as a function of GaAs thickness $n$, calculated by means of the relativistic expression, Eq. (8), but with the SOC suppressed within the $2 m$ -1 inner GaAs layers. Right: The corresponding pessimistic TMR ratio $\left(g^{\mathrm{P}}-g^{\mathrm{AP}}\right) / g^{\mathrm{P}}$. The results for full SOC are also plotted (dashed lines) for comparison (see Ref. 14).

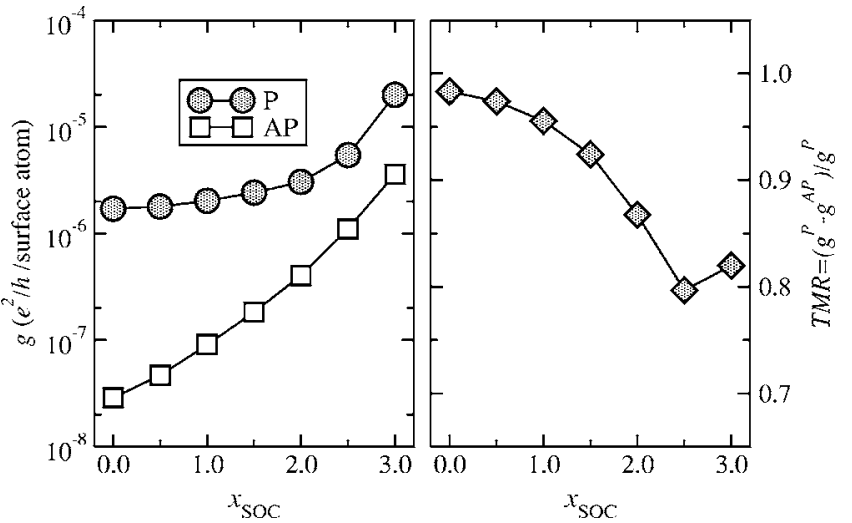

FIG. 5. Left: tunneling conductance for $\mathrm{Fe} / 41(\mathrm{GaAs}) / \mathrm{Fe}$ trilayer system [for parallel (P) and antiparallel (AP) alignment], calculated by means of the relativistic expression, Eq. (8), with the SOC manipulated within the $2 m-1[m=(n-7) / 2=17]$ inner GaAs layers, as a function of spin-orbit interaction strength $x$. Right: The corresponding pessimistic TMR ratio $\left(g^{\mathrm{P}}-g^{\mathrm{AP}}\right) / g^{\mathrm{P}}$.

effect in the two magnetic configurations leads to an increase of the TMR (right panel) through the "scalar-relativistic" spacer, despite the fact that, as mentioned above, SOC suppression does not affect the bottom of the $\mathrm{CB}$ and the height of the tunneling barrier.

We pointed out already that the use of the modified SOC operator, via Eq. (15), is by no means restricted to the particular values $x=0$ or $x=1$ used so far, but allows one to gradually scale up or down the spin-orbit interaction strength. Applying their scheme to transition-metal elements, Ebert et al. ${ }^{15}$ found a linear dependence of the orbital magnetic moments as well as for the Kerr rotation spectra on the scaling parameter $x$. We discuss in the following the dependence of the conductance on the SOC strength. Because of the expression for the conductance, Eq. (8), or, equivalently, for any other response function, involving a product of Green functions, a simple linear dependence is not expected to occur. One can see that this is indeed the case by inspecting the results in Fig. 5. This figure shows the conductance (left) and TMR ratio (right) calculated for the $\mathrm{Fe} / 41 \mathrm{GaAs} / \mathrm{Fe}$ trilayer system with the SOC scaled from $x$ $=0.0$ to $x=3.0$ on the inner $2 m-1=33 \mathrm{Ga}$ and As atoms. For both magnetic configurations an exponential increase of conductance can be observed with increasing $x$, again with a more pronounced impact in the AP configuration. The corresponding TMR ratio decreases parabolically up to $x=2.5$ but begins to oscillate above this value. The reason for this behavior can be understood by analyzing the results in Fig. 6, which shows the $\vec{k}_{\|}$-resolved transmission probability around the $\bar{\Gamma}$ point for several $x$ values. As a general feature, the transmission is enhanced with increasing spin-orbit interaction strength, as expected, throughout the whole 2DBZ. It can also be seen, however, that the transmission channels which are favored are not the same as $x$ varies. This is an obvious indication that the SOC modifies the interference of the different Bloch waves in the spacer.

We recall, on the other hand, that the interference of the tunneling states depends also on the thickness of the barrier. Fluctuations of the TMR ratio were found to occur even at 


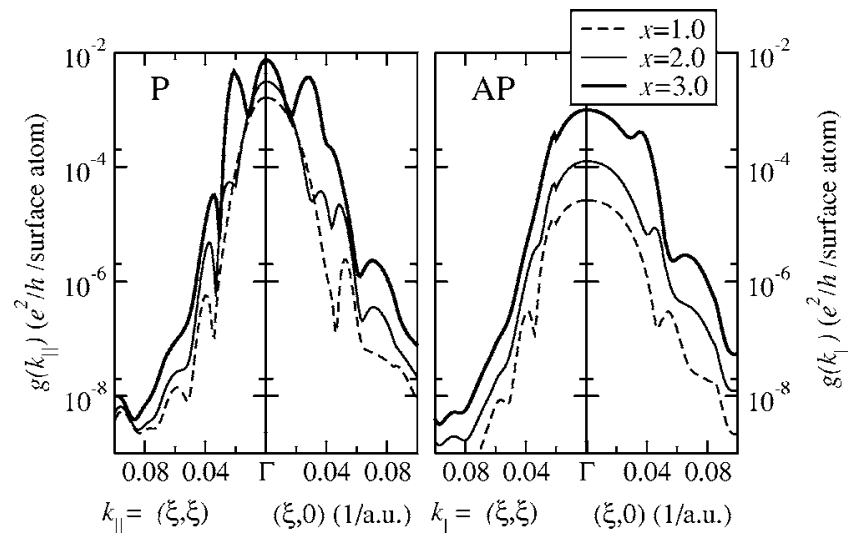

FIG. 6. $\vec{k}_{\|}$-resolved relativistic transmission probability $g\left(\vec{k}_{\|}\right)$, Eq. (9), in Fe/41(GaAs)/Fe along the (110) and (100) directions of the zinc-blende structure, for several values of the spin-orbit interaction strength $x$, which has been modified on the $2 m-1[m=(n$ $-7) / 2=17]$ inner GaAs layers. Left and right side of each panel correspond, respectively, to a parallel (P) and antiparallel (AP) alignment of the magnetization in the two Fe leads.

smaller values of $x$ for thinner spacers, but they are no longer present as the thickness of the barrier is increased. In this case $(n \geqslant 49)$ we could find a monotonous parabolic decay for $x$ values as high as 5.0, far beyond a "super-relativistic" limit. This shows that even in the ballistic limit of a perfect geometry the SOC in the barrier can cause significant changes in the magnetoresistance.

\section{SUMMARY}

We have presented a fully relativistic implementation of the Landauer-Büttiker formula for conductance in the framework of the tight-binding KKR Green-function method. By neglecting the spin off-diagonal matrix elements of the current density operator in the leads we obtained an approximate expression for the conductance which enables, within a relativistic description, a qualitative analysis of the transport according to four terms: two spin-diagonal and two spinmixing transmission channels. The latter ones, originating solely because of the spin-orbit coupling (SOC), should be understood as a shortcut between the former, spin conserving ones.

This formalism has been applied to the case of an $\mathrm{Fe} / n(\mathrm{GaAs}) / \mathrm{Fe}$ trilayer system. The effects induced by including SOC in the calculations have been analyzed and discussed in detail by means of the spin decomposition of conductance. We could show that the most important change caused by adopting a fully relativistic treatment is due to a reduction in spin selectivity of the states involved in the tunneling. Since the spin is not a good quantum number, the states throughout the whole junction have no longer a well defined spin character, and thus an electron entering the junction can occupy either a majority or a minority spin state on the other side.

For a parallel orientation of the magnetization in the $\mathrm{Fe}$ leads the spin mixing induced by SOC is relatively small, leading to about $1 \%$ change for the total transmission. In the antiparallel (AP) alignment, on the other hand, spin-flip channels dominate the transport (up to 90\%) leading to a significant decrease of the magnetoresistance as compared to scalar-relativistic calculations based on the two-current model. Indeed, the effect of SOC should be more pronounced in the AP alignment since majority-spin and minority-spin densities at the Fermi energy have significantly different magnitudes.

An analysis of the $\vec{k}_{\|}$-resolved transmission probability revealed two main aspects concerning the influence of the SOC: in the vicinity of the $\bar{\Gamma}$ point, i.e., close to the normal incidence, the transmission is enhanced because of the aforementioned reduced spin selectivity. Out of normal incidence, SOC has an important role in modifying the interference of the tunneling states, in particular enabling the interface resonant states to decay slower in the barrier.

This particular point was further investigated by means of model calculations. A scheme allowing the manipulation of spin-orbit interaction strength has been applied and two different cases, SOC switched on and off inside the barrier, have been compared and discussed. The changes in transmission induced by SOC have been related to the corresponding changes in the complex electronic band structure of the inner bulklike GaAs layers. As in the case of exact relativistic calculations, a stronger impact on the conductance was found for the AP alignment, in particular on the spin-flip channels. An enhanced value of spin-orbit interaction in the barrier was shown to lead to a further decrease in the magnetoresistance.

Altogether, our investigations have shown that spin-orbit coupling influences the spin-dependent transport both qualitatively and quantitatively and its inclusion in the calculations might be important even for relatively light atoms. Besides, as it was found already both theoretically ${ }^{31}$ and experimentally, ${ }^{32}$ the $\mathrm{Fe}$-zinc-blende interface is characterized by a strong magnetic anisotropy, that is also caused by spin-orbit coupling. A non- or scalar-relativistic transport properties calculation will not distinguish between the magnetization in-plane and out-of-plane geometries since there is no coupling between the spin and orbital degrees of freedom. Therefore corresponding investigations necessarily require a fully relativistic treatment which, combined with a more realistic description of the geometry of the system and accounting for no $\vec{k}_{\|}$-conserving scattering, should arise as a powerful theoretical tool for future investigations.

\section{ACKNOWLEDGMENTS}

This work was supported by the RT Network of Computational Magnetoelectronics of the European Commission. One of us (V.P.) gratefully acknowledges the financial support of the Deutsche Forschungsgemeinschaft within the DFG-Förderprojekt FOG 370/2-1 "Ferromagnet-Halbleiter Nanostrukturen: Transport, elektrische und magnetische Eigenschaften." 
${ }^{1}$ M. Jullière, Phys. Lett. 54A, 225 (1975).

${ }^{2}$ J. C. Slonczewski, Phys. Rev. B 39, 6995 (1989).

${ }^{3}$ J. M. MacLaren, X. G. Zhang, W. H. Butler, and X. Wang, Phys. Rev. B 59, 5470 (1999).

${ }^{4}$ W. H. Butler, X. G. Zhang, X. Wang, J. van Ek, and J. M. MacLaren, J. Appl. Phys. 81, 5518 (1997).

${ }^{5}$ J. M. MacLaren, W. H. Butler, and X. G. Zhang, J. Appl. Phys. 83, 6521 (1998).

${ }^{6}$ E. Tsymbal, O. Mryasov, and P. LeClair, J. Phys.: Condens. Matter 15, R109 (2003).

${ }^{7}$ M. Zwierzycki, K. Xia, P. J. Kelly, G. E. W. Bauer, and I. Turek, Phys. Rev. B 67, 092401 (2003).

${ }^{8}$ O. Wunnicke, P. Mavropoulos, R. Zeller, and P. H. Dederichs, J. Phys.: Condens. Matter 16, 4643 (2004).

${ }^{9}$ C. Zhang, X. G. Zhang, P. S. Krstić, H. P. Cheng, W. H. Butler, and J. M. MacLaren, Phys. Rev. B 69, 134406 (2004).

${ }^{10}$ M. Zenger, J. Moser, S. Kreuzer, W. Wegscheider, D. Weiss, and T. Dietl, J. Appl. Phys. 96, 2400 (2004); M. Zenger, J. Moser, S. Kreuzer, W. Wegscheider, and D. Weiss, J. Phys.: Condens. Matter 16, S5823 (2004).

${ }^{11}$ J. S. Moodera, L. R. Kinder, T. M. Wong, and R. Meservey, Phys. Rev. Lett. 74, 3273 (1995).

${ }^{12}$ H. C. Herper, P. Weinberger, A. Vernes, L. Szunyogh, and C. Sommers, Phys. Rev. B 64, 184442 (2001); P. Weinberger, L. Szunyogh, C. Blaas, and C. Sommers, ibid. 64, 184429 (2001); H. C. Herper, P. Weinberger, L. Szunyogh, and C. Sommers, ibid. 66, 064426 (2002); J. Appl. Phys. 91, 8777 (2002).

${ }^{13}$ J. Henk and P. Bruno, Phys. Rev. B 68, 174430 (2003); H. F. Ding, W. Wulfhekel, J. Henk, P. Bruno, and J. Kirschner, Phys. Rev. Lett. 90, 116603 (2003).

${ }^{14}$ V. Popescu, H. Ebert, N. Papanikolaou, R. Zeller, and P. H. Dederichs, J. Phys.: Condens. Matter 16, S5579 (2004).

${ }^{15}$ H. Ebert, H. Freyer, A. Vernes, and G.-Y. Guo, Phys. Rev. B 53, 7721 (1996).

${ }^{16}$ L. Szunyogh, B. Újfalussy, P. Weinberger, and J. Kollar, Phys. Rev. B 49, 2721 (1994); R. Zeller, P. H. Dederichs, B. Újfalussy, L. Szunyogh, and P. Weinberger, ibid. 52, 8807 (1995);
K. Wildberger, R. Zeller, and P. H. Dederichs, ibid. 55, 10074 (1997).

${ }^{17} \mathrm{H}$. Ebert, in Electronic Structure and Physical Properties of Solids, Lecture Notes in Physics Vol. 535, edited by H. Dreyssé (Springer, Berlin, 2000), p. 191.

${ }^{18}$ M. E. Rose, Relativistic Electron Theory (Wiley, New York, 1961); P. Strange, Relativistic Quantum Mechanics (Cambridge University Press, Cambridge, England, 1998).

${ }^{19}$ H. U. Baranger and A. D. Stone, Phys. Rev. B 40, 8169 (1989).

${ }^{20}$ W. H. Butler, Phys. Rev. B 31, 3260 (1985).

${ }^{21}$ R. Landauer, IBM J. Res. Dev. 1, 223 (1957); M. Büttiker, Phys. Rev. Lett. 57, 1761 (1986); R. Landauer, IBM J. Res. Dev. 32, 306 (1988); M. Büttiker, ibid. 32, 317 (1988).

${ }^{22}$ P. Mavropoulos, N. Papanikolaou, and P. H. Dederichs, Phys. Rev. B 69, 125104 (2004).

${ }^{23}$ X. Wang, X.-G. Zhang, W. H. Butler, G. M. Stocks, and B. N. Harmon, Phys. Rev. B 46, 9352 (1992).

${ }^{24}$ S. H. Vosko, L. Wilk, and M. Nusair, Can. J. Phys. 58, 1200 (1980).

${ }^{25}$ M. Freyss, N. Papanikolaou, V. Bellini, R. Zeller, and P. H. Dederichs, Phys. Rev. B 66, 014445 (2002).

${ }^{26}$ P. Mavropoulos, O. Wunnicke, and P. H. Dederichs, Phys. Rev. B 66, 024416 (2002).

${ }^{27}$ J. Mathon and A. Umerski, Phys. Rev. B 63, 220403(R) (2001).

${ }^{28}$ W. H. Butler, X. G. Zhang, T. C. Schulthess, and J. M. MacLaren, Phys. Rev. B 63, 054416 (2001).

${ }^{29}$ P. Mavropoulos, N. Papanikolaou, and P. H. Dederichs, Phys. Rev. Lett. 85, 1088 (2000).

${ }^{30}$ V. Popescu and H. Ebert (unpublished).

${ }^{31}$ E. Sjöstedt, L. Nordström, F. Gustavsson, and O. Eriksson, Phys. Rev. Lett. 89, 267203 (2002).

${ }^{32}$ F. Bensch, R. Moosbühler, and G. Bayreuther, J. Appl. Phys. 91, 8754 (2002); S. McPhail, C. M. Gurtler, F. Montaigne, Y. B. Xu, M. Tselepi, and J. A. C. Bland, Phys. Rev. B 67, 024409 (2003); M. Madami, S. Tacchi, G. Carlotti, G. Gubbiotti, and R. L. Stamps, ibid. 69, 144408 (2004). 